\title{
HIERARCHY OF INTEGRABLE GEODESIC FLOWS
}

\author{
Peter Topalov
}

Abstract

A family of integrable geodesic flows is obtained. Any such a family corresponds to a pair of geodesically equivalent metrics.

\section{Introduction}

In papers $[\mathbf{1}],[\mathbf{2}],[\mathbf{3}]$ a simple approach was suggested for obtaining first integrals of Hamiltonian systems if a trajectorial diffeomorphism is given. This approach is closely related to the ideas presented in [4].

Recall briefly the main construction (for details see $[\mathbf{1}],[\mathbf{2}]$ ). Let $v$ and $\bar{v}$ be Hamiltonian systems on the symplectic manifolds $\left(M^{2 n}, \omega\right)$ and $\left(\bar{M}^{2 n}, \bar{\omega}\right)$ with Hamiltonians $H$ and $\bar{H}$ respectively. Consider the isoenergy surfaces

$$
Q \stackrel{\text { def }}{=}\left\{x \in M^{2 n} \mid H(x)=h\right\}, \bar{Q} \stackrel{\text { def }}{=}\left\{x \in \bar{M}^{2 n} \mid \bar{H}(x)=\bar{h}\right\},
$$

where $h$ and $\bar{h}$ are regular values of the functions $H$ and $\bar{H}$.

Definition 1. A diffeomorphism $\phi: Q \rightarrow \bar{Q}$ is said to be trajectorial, if it takes the trajectories of the system $v$ to the trajectories of the system $\bar{v}$.

Let $\phi: Q \rightarrow \bar{Q}$ be a trajectorial diffeomorphism. Let us denote the restrictions $\left.\omega\right|_{Q}$ and $\left.\bar{\omega}\right|_{\bar{Q}}$ also by the letters $\omega$ and $\bar{\omega}$. Then the pullback vanish (of course, also $L_{\bar{v}} \omega=0$ ). It is obvious that the kernels of the forms $\omega$ and $\phi^{*} \bar{\omega}$ coincide with the linear span of the vector $v$. Therefore, these forms induce two non-degenerate tensor fields on the quotient bundle $\mathcal{T} Q /\langle v\rangle$. Thus, the characteristic polynomial of the operator $\omega^{-1} \circ \phi^{*} \bar{\omega}$ is preserved by the flow $v$.

In papers $[\mathbf{2}],[\mathbf{3}]$ the construction was applied to a classical example where such a diffeomorphism exists - the geodesic flows corresponding to a pair of geodesically equivalent metrics.

Let $g$ and $\bar{g}$ be Riemannian metrics on the manifold $M^{n}$. 
Definition 2. The pseudo-Riemannian metrics $g$ and $\bar{g}$ are called geodesically equivalent iff they have the same geodesics (considered as unparameterized curves).

For geodesically equivalent Riemannian metrics, a trajectorial diffeomorphism $\Phi$ is given by the formula $\Phi:(x, \xi) \mapsto\left(x, \frac{\|\xi\|_{g}}{\|\xi\|_{\bar{g}}} \xi\right)$, where $\xi \in \mathcal{T}_{x} M^{n}$.

Denote by $\mathcal{M}^{q}(g, \bar{g})(1 \leq q \leq n)$ the set of points $y \in M^{n}$ such that the Riemannian metrics $g$ and $\bar{g}$ have exactly $q$ distinct eigenvalues in an open neighborhood of the point $y$. If the set $\mathcal{M}^{n}$ is everywhere dense in $M^{n}$ we say that the Riemannian metrics $g$ and $\bar{g}$ are strictly non-proportional on $M^{n}$.

In the papers $[\mathbf{2}],[\mathbf{3}]$ the following was proved.

Theorem 1. Suppose the Riemannian metrics $g$ and $\bar{g}$ are geodesically equivalent; then

a) the geodesic flow of the metric $g$ admits $n$ integrals $(k \geq 0)$

$$
I_{k} \stackrel{\text { def }}{=}(-1)^{k}\left(\frac{\operatorname{det}(g)}{\operatorname{det}(\bar{g})}\right)^{\frac{k+2}{n+1}} \bar{g}\left(S_{k} v, v\right)
$$

where

$$
S_{k} \stackrel{\text { def }}{=} \bar{G}^{k}-\sigma_{1}(\bar{G}) \bar{G}^{k-1}+\cdots+(-1)^{k} \sigma_{k}(\bar{G})
$$

and $\bar{G} \stackrel{\text { def }}{=}\left(g^{i j} \bar{g}_{j k}\right), \sigma_{i}(\bar{G})$ are the elementary symmetric polynomials of degree $i$;

b) the integrals $I_{k}(k \geq 0)$ are in involution ${ }^{1}$;

c) if $\mathcal{M}^{q} \neq \emptyset$, then the rank of the differentials $d I_{0}, \ldots, d I_{n-1}$ is equal to $q$ almost everywhere in $\mathcal{T} \mathcal{M}^{q}$. Moreover, on $\mathcal{T} \mathcal{M}^{q}$ we have

$$
\operatorname{rk}\left(d I_{0}, \ldots, d I_{n-1}\right)=\operatorname{rk}\left(d I_{0}, \ldots, d I_{q-1}\right) \leq q .
$$

This theorem is closely related to some results proved by U. Dini, P. Painlevé, T. Levi-Civita and R. Liouville (see [5], [6]).

In the present paper we assign to any pair of geodesically equivalent metrics a hierarchy of integrable geodesic flows (Theorem 5 ). The paper is organized as follows.

In Section 2 we present some important facts from the theory of geodesic mappings needed for the sequel.

In Section 3 we assign to any pair of strictly non-proportional geodesically equivalent metrics $g$ and $\bar{g}$ a family of completely integrable

${ }^{1}$ I.e., making the Legendre transformation we obtain $n$ functions commuting with respect to the canonical symplectic structure on $\mathcal{T}^{*} M$. 
Riemannian metrics $\mathcal{S}(g, \bar{g})$ (see Theorem 5). Some simple properties of these families are obtained.

In Section 4 the results obtained in Section 3 are applied to the metrics

$$
d g^{2} \stackrel{\text { def }}{=} \sum_{i=1}^{n} d x^{i^{2}}
$$

and

$$
d \bar{g}^{2} \stackrel{\text { def }}{=} \frac{1}{\sum_{i=1}^{n}\left(\frac{x^{i}}{a^{i}}\right)^{2}} \sum_{i=1}^{n} \frac{d x^{i^{2}}}{a^{i}}
$$

which are geodesically equivalent on the standard ellipsoid $E_{h} \stackrel{\text { def }}{=}$ $\left\{\sum_{i=1}^{n} \frac{x^{i^{2}}}{a^{i}}=h\right\}, h>0$. We give explicit formulae for the family $\mathcal{S}\left(\left.d g^{2}\right|_{E_{h}},\left.d \bar{g}^{2}\right|_{E_{h}}\right)$ in terms of some metrics on the whole $R^{n}$ (Theorem 6). It is interesting that this family contains the metric on the Poisson sphere, well-known in classical mechanics. It immediately permits us to prove that the geodesic flow on the standard ellipsoid and the geodesic flow on the Poisson sphere have the same Liouville foliation (Corollary 3). This theorem is a multidimensional generalization of the well-known one in the two-dimensional case. In addition, we give an explicit formula for a metric geodesically equivalent to the metric on the Poisson sphere (Corollary 2).

In Section 5 we obtain a family of completely integrable Hamiltonians with respect to the standard Lie-Poisson bracket on $e(3)^{*}$ (the dual space to the Lie algebra $e(3))$. In particular, we obtain an algebraic Hamiltonian $H^{(1)}$ such that the corresponding Hamiltonian system sgrad $H^{(1)}$ is orbitally equivalent to the Euler case of the free motion of the rigid body (see Corollary 6 ).

Throughout the paper the standard agreement holds, i.e. Riemannian metric means a positive-definite symmetric form and pseudo-Riemannian metric means a non-degenerate symmetric form. We consider mainly Riemannian metrics; nevertheless many results hold in the pseudoRiemannian case.

The author is grateful to A. V. Bolsinov, A. T. Fomenko, V. V. Kozlov, V. S. Matveev, S. Tabachnikov and I. A. Taimanov for useful discussions. This paper was written during my stay at the Max-Planck-Institut für Mathematik in Bonn. It is pleasure to thank the Institut for its hospitality and financial support. The author is partially supported by MESC grant MM-810/98. 


\section{Geodesically equivalent metrics and the corresponding 1-parameter family}

The following lemma is needed for the sequel.

Lemma 1 (see [8]).

1) Suppose the pseudo-Riemannian metrics $g$ and $\bar{g}$ are geodesically equivalent; then the tensors $a_{i j}$ and $\lambda_{i}$

$$
\begin{aligned}
a_{i j} & \stackrel{\text { def }}{=} A_{i}^{\alpha} g_{\alpha j}, \\
\lambda_{i} & \stackrel{\text { def }}{=}-A_{i}^{\alpha} \psi_{\alpha},
\end{aligned}
$$

where $(n+1) \psi_{i}=\frac{1}{2} \partial_{i} \ln \left|\frac{\bar{g}}{g}\right|, g=\operatorname{det}\left(g_{i j}\right)$, and the operator $A$ is given by formula

$$
A_{j}^{i}(g, \bar{g}) \stackrel{\text { def }}{=}\left|\frac{\bar{g}}{g}\right|^{\frac{1}{n+1}} \bar{g}^{i \alpha} g_{\alpha j},
$$

satisfy the equation

$$
a_{i j, k}=\lambda_{i} g_{j k}+\lambda_{j} g_{i k} .
$$

Here $a_{i j, k}$ denotes the covariant derivative $\nabla_{k} a_{i j}$, where $\nabla$ is the Levi-Civita connection corresponding to the metric $g$.

2) Conversely, if a non-degenerate symmetric tensor field $a_{i j}$ and an 1-form $\lambda_{i}$ satisfy equation (9), then the metric

$$
\bar{g}_{i j} \stackrel{\text { def }}{=}\left(\frac{\hat{g}}{g}\right) \hat{g}_{i j}
$$

where $\hat{g}_{i j} \stackrel{\text { def }}{=} g_{i \alpha} a^{\alpha \beta} g_{\beta j}$, is geodesically equivalent to $g$.

It is obvious that $A$ is self-adjoint with respect the both metrics.

Now, using Lemma 1 we can prove

Proposition 1. Suppose the pseudo-Riemannian metrics $g$ and $\bar{g}$ are geodesically equivalent; then if for some parameters $\alpha$ and $\beta$ the operator $(\alpha A+\beta)$ is invertible on $M^{n}$, then the pseudo-Riemannian metrics $g$ and

$$
\bar{g}_{\alpha, \beta}(X, Y) \stackrel{\text { def }}{=} \frac{1}{\operatorname{det}(\alpha A+\beta)} g\left((\alpha A+\beta)^{-1} X, Y\right),
$$

are geodesically equivalent.

Remark 1. Remark here that $\bar{g}_{0,1}=g$ and $\bar{g}_{1,0}=\bar{g}$. 
Remark 2. If the manifold $M^{n}$ is compact, then formula (11) gives a 1-parameter family of geodesically equivalent metrics. Moreover, in a small neighborhood of each point of $M^{n}$ we also obtain 1-parameter family.

Remark 3. Let us take two representatives $\bar{g}_{\alpha, \beta}$ and $\bar{g}_{\lambda, \mu}$. Now, we are able to apply Proposition 1 again. The corresponding family of geodesically equivalent metrics is

$$
\bar{g}_{a, b}=\frac{1}{\operatorname{det}((a \lambda+b \alpha) A+(a \mu+b \beta))} g((a \lambda+b \alpha) A+(a \mu+b \beta))^{-1} .
$$

Therefore, if $(\alpha: \beta) \neq(\lambda: \mu)$, then we obtain the same family. Remark here also that

$$
A\left(\bar{g}_{\alpha, \beta}, \bar{g}_{\lambda, \mu}\right)=\frac{\lambda A+\mu}{\alpha A+\beta} .
$$

Proof of Proposition 1: Let $g$ and $\bar{g}$ be geodesically equivalent metrics. Using (6), we obtain that the symmetric form $a=g A$ satisfies equation (9) for some $\lambda$. Hence, the form $a_{c} \stackrel{\text { def }}{=} g A+c g(c=$ const $)$ also satisfied this equation. We use here that $\nabla$ is the Levi-Civita connection corresponding to the metric $g$. Assume that $a_{c}$ is non-degenerate on $M^{n}$. We get $\hat{g} \stackrel{\text { def }}{=} g(g A+c g)^{-1} g=g(A+c)^{-1}$ and using the converse formula (10) obtain that the pseudo-Riemannian metric

$$
\bar{g}_{c}=\frac{1}{\operatorname{det}(A+c)} g(A+c)^{-1}
$$

is geodesically equivalent to $g$.

Now, using Proposition 1 we are able to present another 'non-symplectic' proof of the first item of Theorem 1 .

Corollary 1. If the Riemannian metrics $g$ and $\bar{g}$ are geodesically equivalent, then the geodesic flow of the metric $g$ admits a 1-parameter family of first integrals

$$
I_{\alpha, \beta}(g, \bar{g}) \stackrel{\text { def }}{=} \operatorname{det}(\alpha A+\beta) g(\alpha A+\beta)^{-1} .
$$

Proof of Corollary 1: The corollary easily follows from the next theorem (see $[6])$. 
Theorem 2 (Painlevé). If the metrics $g$ and $\bar{g}$ are geodesically equivalent, then the function

$$
I_{0}(g) \stackrel{\operatorname{def}}{=}\left(\frac{\operatorname{det}(g)}{\operatorname{det}(\bar{g})}\right)^{\frac{2}{n+1}} \bar{g}
$$

is an integral of the the geodesic flow of the metric $g$.

In a small neighborhood of each point of $M^{n}$ we can find a 1-parameter family $\bar{g}_{1, \beta}$ of geodesically equivalent Riemannian metrics. Therefore, locally the Riemannian metrics $g$ and $\bar{g}_{1, \beta}$ are geodesically equivalent, where $\beta \in(a, b), a<b$. It permits us to apply Theorem 2 . We have

$$
\begin{aligned}
I_{1, \beta}(g) & \stackrel{\text { def }}{=}\left(\frac{\operatorname{det}(g)}{\operatorname{det}\left(\bar{g}_{1, \beta}\right)}\right)^{\frac{2}{n+1}} \bar{g}_{1, \beta} \\
& =\operatorname{det}(A+\beta) g(A+\beta)^{-1} \\
& =I_{0}+I_{1} \beta+\cdots+I_{n-1} \beta^{n-1},
\end{aligned}
$$

where the functions $I_{k}$ are the same as in Theorem 1. The last equality in this chain can easily be proved. Therefore, the functions $I_{k}$ are integrals in a small neighborhood of each point of $M^{n}$. But they are globally defined on $M^{n}$. Thus, they are integrals.

Remark 4. If we apply Corollary 1 to the geodesically equivalent metrics $g$ and $\bar{g}_{\alpha, \beta}((\alpha: \beta) \neq(0: 1))$, then we'll obtain the same family of integrals.

Remark 5. Let us consider the map $\Phi: \xi \mapsto \frac{\|\xi\|_{g}}{\|\xi\|_{\bar{g}}} \xi^{2}$,

$$
\Phi:(\mathcal{T} M)_{0} \rightarrow(\mathcal{T} M)_{0} .
$$

It is obvious that $\Phi$ maps each geodesic trajectory of the metric $g$ into a geodesic trajectory of the metric $\bar{g}$. Therefore, the pull-back $\Phi^{*}\left(I_{\alpha, \beta}(\bar{g}, g)\right)$ gives a family of integrals for the geodesic flow of the metric $g$. It can easily be checked that

$$
\Phi^{*}\left(I_{\alpha, \beta}(\bar{g}, g)\right)=\frac{g(\xi, \xi)}{I_{1,0}(g, \bar{g})} I_{\beta, \alpha}(g, \bar{g}) .
$$

Thus, we won't be able to obtain new family of integrals.

${ }^{2}$ Sometimes we will denote this mapping by $\Phi(g, \bar{g})$. 


\section{Hierarchy of integrable flows}

As we have seen if the Riemannian metrics $g$ and $\bar{g}$ are geodesically equivalent, then they are contained in a family of geodesically equivalent metrics

$$
\bar{g}_{\alpha, \beta}=\frac{1}{\operatorname{det}(\alpha A+\beta)} g(\alpha A+\beta)^{-1},
$$

where $\alpha$ and $\beta$ are parameters.

Remark 6 . In the following sections we consider only Riemannian metrics although the most of the constructions pass in pseudo-Riemannian case.

It is interesting that using geodesically equivalent Riemannian metrics $g$ and $\bar{g}$ we can produce another families of geodesically equivalent metrics. We need

Theorem 3 (see $[\mathbf{8}]$ ). If the Riemannian metrics $g$ and $\bar{g}$ are geodesically equivalent, then for each integer $k$ the Riemannian metrics $g(k) \stackrel{\text { def }}{=}$ $g A^{k}$ and $\bar{g}^{(k)} \stackrel{\text { def }}{=} \bar{g} A^{k}$ are also geodesically equivalent.

This theorem may be proved by direct calculations. For details see paper $[\mathbf{8}]$. We will slightly generalize this result later using some other arguments. At first we need some notations.

Let $B$ be a self-adjoint operator on the connected Riemannian manifold $\left(M^{n}, g\right)$. By definition, put

$$
r(B) \stackrel{\text { def }}{=} \inf _{x \in M} \min \{\operatorname{spec} B(x)\}
$$

and

$$
R(B) \stackrel{\text { def }}{=} \sup _{x \in M} \max \{\operatorname{spec} B(x)\}
$$

Denote by $I$ the set obtained by adding to the interval $(r, R)$ its endpoints iff they are achieved for some $x \in M^{n}$. Let us consider the set of all real Laurent series $L_{a}(x)=\sum_{k} c_{k}(x-a)^{k}$ which are convergent on some open neighborhood of $I$. Denote by $\omega^{+}(B)$ the cone of all finite linear combinations of such series which give positive functions on $I$. Of course, we are able to consider more general set of functions but it will only complicate our construction. 
Theorem 4. Suppose the Riemannian metrics $g$ and $\bar{g}$ are geodesically equivalent and $F(x) \in \omega^{+}(A)$; then the metrics $g^{F} \stackrel{\text { def }}{=} g F(A)$ and $\bar{g}^{F} \stackrel{\text { def }}{=}$ $\bar{g} F(A)$ are also geodesically equivalent. These metrics are contained in a family of geodesically equivalent Riemannian metrics

$$
\bar{g}_{\alpha, \beta}^{F} \stackrel{\text { def }}{=} \frac{1}{\operatorname{det}(\alpha A+\beta)} g F(A)(\alpha A+\beta)^{-1},
$$

where $\alpha$ and $\beta$ are parameters such that the operator $(\alpha A+\beta)$ is nondegenerate.

Proof of Theorem 4: Let $g$ and $\bar{g}$ be geodesically equivalent Riemannian metrics on a manifold $M^{n}$. Denote by $\rho^{1}, \ldots, \rho^{m}(1 \leq m \leq n)$ the common eigenvalues of the metrics $g$ and $\bar{g}$. Suppose the functions $\rho^{1}, \ldots, \rho^{m}$ are different at every point of an open domain $\mathcal{D} \subset M^{n}$. In the paper [6], T. Levi-Civita proved that for every point $P \in \mathcal{D}$ there is an open neighborhood $\mathcal{U}(P) \subset \mathcal{D}$ and a coordinate system $\bar{x}=\left(\bar{x}_{1}, \ldots, \bar{x}_{m}\right)($ in $\mathcal{U}(P))$, where $\bar{x}_{i}=\left(x_{i}^{1}, \ldots, x_{i}^{k_{i}}\right),(1 \leq i \leq m)$, such that the quadratic forms of the metrics $g$ and $\bar{g}$ have the following form:

$$
\begin{aligned}
& g(\dot{\bar{x}}, \dot{\bar{x}})=\sum_{i=1}^{m} \Pi_{i}(\bar{x}) A_{i}\left(\bar{x}_{i}, \dot{\bar{x}}_{i}\right), \\
& \bar{g}(\dot{\bar{x}}, \dot{\bar{x}})=\sum_{i=1}^{m} \rho^{i} \Pi_{i}(\bar{x}) A_{i}\left(\bar{x}_{i}, \dot{\bar{x}}_{i}\right),
\end{aligned}
$$

where $A_{i}\left(\bar{x}_{i}, \dot{\bar{x}}_{i}\right)$ are positive-definite quadratic forms in the velocities $\dot{\bar{x}}_{i}$ with coefficients depending on $\bar{x}_{i}$,

$$
\begin{gathered}
\Pi_{i} \stackrel{\text { def }}{=}\left(\phi_{i}-\phi_{1}\right) \ldots\left(\phi_{i}-\phi_{i-1}\right)\left(\phi_{i+1}-\phi_{i}\right) \ldots\left(\phi_{m}-\phi_{i}\right), \\
\rho^{i}=\frac{1}{\phi_{1} \ldots \phi_{m}} \frac{1}{\phi_{i}}
\end{gathered}
$$

and $\phi_{1}, \phi_{2}, \ldots, \phi_{m}\left(0<\phi_{1}<\phi_{2}<\cdots<\phi_{m}\right)$ are smooth functions such that

$$
\phi_{i}= \begin{cases}\phi_{i}\left(\bar{x}_{i}\right), & \text { if } k_{i}=1 \\ \text { constant, } & \text { else. }\end{cases}
$$


Definition 3. Let metrics $g$ and $\bar{g}$ be given by formulae (26) and (27) in a coordinate chart $\mathcal{U}$. Then we say that the metrics $g$ and $\bar{g}$ have LeviCivita local form of (type $m$ ), and the coordinate chart $\mathcal{U}$ is Levi-Civita coordinate chart (with respect to the metrics).

In the paper [6], Levi-Civita proved that the metrics $g$ and $\bar{g}$ given by formulae (26) and (27) are geodesically equivalent.

Denote by $\mathcal{M}$ the set of all point in $M^{n}$ which are contained in some Levi-Civita chart, i.e. $x \in \mathcal{M}$ iff there is a Levi-Civita chart $\mathcal{U}$ which contains $x$. By definition, $\mathcal{M}$ is an open subset of $M^{n}$. In [2], [3] was proved that $\mathcal{M}$ is everywhere dense in $M^{n}$.

In every Levi-Civita coordinate chart we have

$$
\begin{aligned}
\left(\frac{\bar{g}}{g}\right)^{\frac{1}{n+1}}= & {\left[\frac{1}{\phi_{1}^{k_{1}-1} \ldots \phi_{m}^{k_{m}-1}}\right] \frac{1}{\phi_{1} \ldots \phi_{m}}=\text { const. } \frac{1}{\phi_{1} \ldots \phi_{m}}, } \\
\left(\bar{g}^{i k} g_{k j}\right) & =\operatorname{diag}(\underbrace{\frac{1}{\rho^{1}}, \ldots, \frac{1}{\rho^{1}}}_{k_{1}} ; \ldots ; \underbrace{\frac{1}{\rho^{m}}, \ldots, \frac{1}{\rho^{m}}}_{k_{m}}) .
\end{aligned}
$$

Therefore,

$$
A(g, \bar{g})=\text { const. } \operatorname{diag}(\underbrace{\phi^{1}, \ldots, \phi^{1}}_{k_{1}} ; \ldots ; \underbrace{\phi^{m}, \ldots, \phi^{m}}_{k_{m}}) .
$$

Hence,

$$
g(F(A) \dot{\bar{x}}, \dot{\bar{x}})=\sum_{i=1}^{m} \Pi_{i}(\bar{x}) A_{i}^{\prime}\left(\bar{x}_{i}, \dot{\bar{x}}_{i}\right)
$$

and

$$
\bar{g}(F(A) \dot{\bar{x}}, \dot{\bar{x}})=\sum_{i=1}^{m} \rho^{i} \Pi_{i}(\bar{x}) A^{\prime}{ }_{i}\left(\bar{x}_{i}, \dot{\bar{x}}_{i}\right),
$$

where $A^{\prime}{ }_{i}=f_{i}\left(\bar{x}_{i}\right) A_{i}$ and $f_{i}\left(\bar{x}_{i}\right)$ is a smooth function depending of the variable $\bar{x}_{i}$. Therefore, in any Levi-Civita chart the metrics $g F(A)$ and $\bar{g} F(A)$ are geodesically equivalent. Let us consider the map $\Phi: \xi \mapsto$ $\frac{\|\xi\|_{g}}{\|\xi\|_{\bar{g}}} \xi$

$$
\Phi:(\mathcal{T} M)_{0} \rightarrow(\mathcal{T} M)_{0}
$$


Denote by $v$ and $\bar{v}$ the vector fields corresponding to the geodesic flows of the metrics $g F(A)$ and $\bar{g} F(A)$. Therefore, the smooth vector fields $\bar{v} \neq 0$ and $\Phi_{*}(v) \neq 0$ are proportional on a dense subset of the iso-energetic surface $U^{r} M,(r>0)$. Thus, their directions coincide everywhere. It yields that the metrics $g F(A)$ and $\bar{g} F(A)$ are geodesically equivalent.

Finally, using Proposition 1, we obtain a family of geodesically equivalent metrics

$$
\begin{aligned}
\bar{g}_{\alpha, \beta}^{F} & \stackrel{\text { def }}{=} \frac{1}{\operatorname{det}\left(\alpha A\left(g^{F}, \bar{g}^{F}\right)+\beta\right)} g^{F}\left(\alpha A\left(g^{F}, \bar{g}^{F}\right)+\beta\right)^{-1} \\
& =\frac{1}{\operatorname{det}(\alpha A+\beta)} g^{F}(\alpha A+\beta)^{-1} .
\end{aligned}
$$

This completes the proof of Theorem 4 .

If the Riemannian metrics $g$ and $\bar{g}$ are strictly non-proportional geodesically equivalent on $M^{n}$, then the metrics $g^{F}$ and $\bar{g}^{F}$ are also strictly non-proportional geodesically equivalent. Therefore, using Theorem 1, we obtain that all Riemannian metrics $\bar{g}_{\alpha, \beta}^{F}$ are completely integrable.

Theorem 5. If $g$ and $\bar{g}$ are strictly non-proportional geodesically equivalent Riemannian metrics, then the family of Riemannian metrics

$$
S(g, \bar{g}) \stackrel{\text { def }}{=}\left\{\bar{g}_{\alpha, \beta}^{F} \mid F(x) \in \omega^{+}(A), \alpha, \beta \text { are parameters }\right\}
$$

consists of completely integrable Riemannian metrics.

Remark 7. An explicit formula for the integrals of the metric $g^{F}$ is

$$
\begin{aligned}
I_{\alpha, \beta}\left(g^{F}, \bar{g}^{F}\right) & =\operatorname{det}(\alpha A+\beta) g F(A)(\alpha A+\beta)^{-1} \\
& =\left(\frac{g}{\bar{g}}\right)^{\frac{2}{n+1}} I_{\beta, \alpha}\left(\bar{g}^{F}, g^{F}\right) .
\end{aligned}
$$

Definition 4. The family $S(g, \bar{g})$ is called $S$-hierarchy of integrable Riemannian metrics corresponding to the pair $g$ and $\bar{g}$.

Remark 8. The family $S(g, \bar{g})$ in some sense is 'complete'. It means that if we take two representatives $\bar{g}_{\alpha, \beta}^{F}$ and $\bar{g}_{\lambda, \mu}^{F}((\alpha: \beta) \neq(\lambda: \mu))$, then $S\left(\bar{g}_{\alpha, \beta}^{F}, \bar{g}_{\lambda, \mu}^{F}\right)=S(g, \bar{g})$.

Now, we are going to establish some relations between the flows of the metrics of a given $S$-hierarchy. 
Proposition 2. The metrics from a given S-hierarchy have identical 'Liouville foliations'. It means that for every two metrics $g_{1}$ and $g_{2}$ we can find

(i) $n$ independent almost everywhere on $(\mathcal{T} M)_{0}$ pairwise commuting integrals $\left\{I_{k}^{(1)}\right\}_{k=0}^{n-1}$ for the geodesic flow of the metric $g_{1}$;

(ii) $n$ independent almost everywhere on $(\mathcal{T} M)_{0}$ pairwise commuting integrals $\left\{I_{k}^{(2)}\right\}_{k=0}^{n-1}$ for the geodesic flow of the metric $g_{2}$;

(iii) a diffeomorphism $\phi:(\mathcal{T} M)_{0} \rightarrow(\mathcal{T} M)_{0}$,

such that $\phi^{*} I_{k}^{(2)}=I_{k}^{(1)}$.

Proof of Proposition 2: Suppose $g_{1}, g_{2} \in S(g, \bar{g})$. Without loss of generality it can be assumed that $g_{1}=g$ and $g_{2}=\bar{g}_{\alpha, \beta}^{G}$. Let us consider the commutative diagram

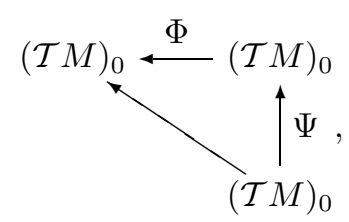

where $\Phi \stackrel{\text { def }}{=} \Phi\left(g^{G}, \bar{g}_{\alpha, \beta}^{G}\right)$ and $\Psi: \xi \rightarrow(\sqrt{G(A)})^{-1} \xi$. Let $\phi \stackrel{\text { def }}{=} \Phi \circ \Psi$. Now, the proposition easily follows from Remark 5 and Remark 7 .

Let $g$ and $\bar{g}$ be strictly non-proportional geodesically equivalent Riemannian metrics on the manifold $M^{n}$. Consider the diagram

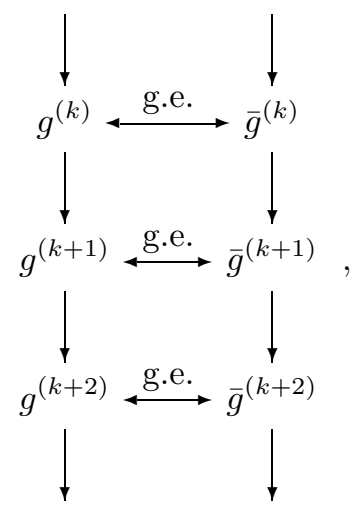

where the horizontal arrows mean that the corresponding Riemannian metrics are geodesically equivalent. Recall that $g^{(k+1)}=g^{(k)} A$, where 
$A \stackrel{\text { def }}{=} A(g, \bar{g})$. We proved that all metrics of the above sequence are completely integrable.

Proposition 3. Let us consider the Riemannian metrics $g^{(k)}$ and $\bar{g}^{(k+2)}$. Making the corresponding Legendre transformations we obtain two Hamiltonian systems on the cotangent bundle $\mathcal{T}^{*} M$ which are completely integrable and the corresponding integrals, canonically given by Theorem 1, coincide.

Proof of Proposition 3: Making the Legendre transformations we obtain two families of pairwise commuting functions on $\mathcal{T}^{*} M$

a) $I_{\alpha, \beta}\left(g^{(k)}, \bar{g}^{(k)}\right)=\operatorname{det}(\alpha A+\beta)(\alpha A+\beta)^{-1}\left(g A^{k}\right)^{-1}$;

b) $I_{\alpha, \beta}\left(\bar{g}^{(k)}, g^{(k)}\right)=\operatorname{det}\left(\alpha A^{-1}+\beta\right)\left(\alpha A^{-1}+\beta\right)^{-1}\left(\bar{g} A^{k}\right)^{-1}$.

We obviously have

$$
\begin{aligned}
I_{\alpha, \beta}\left(\bar{g}^{(k)}, g^{(k)}\right) & =\frac{|\alpha+\beta A|}{|A|}\left[\left(\alpha A^{-1}+\beta\right)^{-1} A^{-1}\right] A^{-k+2}\left[A^{-1} \bar{g}^{-1}\right] \\
& =\left[\frac{1}{|A|}\left(\frac{g}{\bar{g}}\right)^{\frac{1}{n+1}}\right]\left(\operatorname{det}(\alpha+\beta A)(\alpha+\beta A)^{-1} A^{-k+2} g^{-1}\right. \\
& =I_{\beta, \alpha}\left(g^{(k-2)}, \bar{g}^{(k-2)}\right) .
\end{aligned}
$$

This completes the proof.

\section{S-hierarchy for the ellipsoid}

In $[\mathbf{2}]$ was proved that the restrictions of the metrics

$$
d g^{2} \stackrel{\text { def }}{=} \sum_{i=1}^{n} d x^{i^{2}}
$$

and

$$
d \bar{g}^{2} \stackrel{\text { def }}{=} \frac{1}{\sum_{i=1}^{n}\left(\frac{x^{i}}{a^{i}}\right)^{2}} \sum_{i=1}^{n} \frac{d x^{i^{2}}}{a^{i}}
$$

to the ellipsoid $E_{h} \stackrel{\text { def }}{=}\left\{\sum_{i=1}^{n} \frac{x^{i^{2}}}{a^{i}}=h\right\}, h>0$ are geodesically equivalent $^{3}$. Moreover, these metrics are strictly non-proportional on the ellipsoid. Without loss of generality we can assume that $a^{1}<a^{2}<\cdots<$ $a^{n}$.

${ }^{3}$ The same result was independently obtained by S. Tabachnikov in $[\mathbf{9}]$. 
Our aim in the present section is to find explicit formulae for $S$ hierarchy $\mathcal{S}(g, \bar{g})$. Moreover, we intend to extend these metrics on the whole $R^{n}$ in a natural manner.

By definition, put $A \stackrel{\text { def }}{=} \operatorname{diag}\left(a^{1}, \ldots, a^{n}\right)$. Denote by $\langle.,$.$\rangle the natural$ pairing in $R^{n}$.

Let us consider two smooth operators on $R^{n} \backslash\{0\}$

$$
\mathcal{A}(x) \stackrel{\text { def }}{=} A-\frac{1}{\left\langle A^{-1} x, x\right\rangle} x \otimes x+\left(A^{-1} x\right) \otimes\left(A^{-1} x\right)
$$

and

$$
\mathcal{A}^{\prime}(x) \stackrel{\text { def }}{=} A^{-1}-\frac{\left(A^{-1} x\right) \otimes\left(A^{-2} x\right)+\left(A^{-2} x\right) \otimes\left(A^{-1} x\right)}{\left\langle A^{-1} x, A^{-1} x\right\rangle}
$$

where $x=\left(x^{1}, \ldots, x^{n}\right)$.

Proposition 4. The operators $\mathcal{A}$ and $\mathcal{A}^{\prime}$ satisfy the following conditions:

1) both of them are self-adjoint with respect to the metric g;

2) for each $x \in R^{n}$ the tangent space $T_{x} E_{h}$ is invariant subspace for these operators;

3) $\mathcal{A}(x)\left(A^{-1} x\right)=\left\langle A^{-1} x, A^{-1} x\right\rangle A^{-1} x$ and

$$
\mathcal{A}^{\prime}(x)\left(A^{-1} x\right)=-\frac{\left\langle A^{-2} x, A^{-1} x\right\rangle}{\left\langle A^{-1} x, A^{-1} x\right\rangle} A^{-1} x ;
$$

4) the restriction of the operator $\mathcal{A}(x)$ to each ellipsoid $E_{h}, h>0$ coincides (up to multiplication on a positive constant) with the operator $A\left(\left.g\right|_{E_{h}},\left.\bar{g}\right|_{E_{h}}\right)$ and the restriction of $\mathcal{A}^{\prime}$ coincides (up to multiplication on a positive constant) with $\left[A\left(\left.g\right|_{E_{h}},\left.\bar{g}\right|_{E_{h}}\right)\right]^{-1}$.

These conditions determine the operators $\mathcal{A}$ and $\mathcal{A}^{\prime}$ uniquely.

Proof of Proposition 4: Items 1) and 3) are obvious.

Let us prove 2). Suppose that $\xi \in T_{x} E_{h}$, i.e. $\left\langle A^{-1} x, \xi\right\rangle=0$. We have

$$
\begin{aligned}
\left\langle A^{-1} x, \mathcal{A}(x)(\xi)\right\rangle & =\left\langle A^{-1} x, A \xi-\frac{\langle x, \xi\rangle}{\left\langle A^{-1} x, x\right\rangle} x\right\rangle \\
& =\langle x, \xi\rangle-\langle x, \xi\rangle=0
\end{aligned}
$$

and

$$
\begin{aligned}
\left\langle A^{-1} x, \mathcal{A}^{\prime}(x)(\xi)\right\rangle & =\left\langle A^{-1} x, A^{-1} \xi-\frac{\left\langle A^{-2} x, \xi\right\rangle}{\left\langle A^{-1} x, A^{-1} x\right\rangle} A^{-1} x\right\rangle \\
& =\left\langle A^{-1} x, A^{-1} \xi\right\rangle-\left\langle A^{-2} x, \xi\right\rangle=0 .
\end{aligned}
$$

Before proving 4 ) we need the next simple lemma. 
Lemma 2. Let $V$ be an oriented real vector space and $T \subset V$ be an oriented hyperplane. If $g$ and $\bar{g}$ are positive defined metrics, then

$$
\frac{\operatorname{vol}\left(\left.\bar{g}\right|_{T}\right)}{\operatorname{vol}\left(\left.g\right|_{T}\right)}=\frac{\operatorname{vol}(\bar{g})}{\operatorname{vol}(g)} g\left(n_{g}, n_{\bar{g}}\right),
$$

where $n_{g}$ and $n_{\bar{g}}$ are positive directed unit normals to the subspace $T$ calculated for the metrics $g$ and $\bar{g}$ correspondingly.

The proof of Lemma 2 is trivial.

Now, using the lemma, we obtain

$$
\left[\frac{\operatorname{det}\left(\left.\bar{g}\right|_{T_{x}}\right)}{\operatorname{det}\left(\left.g\right|_{T_{x}}\right)}\right]^{\frac{1}{(n-1)+1}}=\frac{\left\langle A^{-1} x, x\right\rangle^{\frac{1}{n}}}{\left(\Pi_{i=1}^{n} a^{i}\right)^{\frac{1}{n}}} \frac{1}{\left\langle A^{-1} x, A^{-1} x\right\rangle} .
$$

If $\xi, \eta \in T_{x} E_{h}$, then we get

$$
\begin{aligned}
\bar{g}_{x}(\mathcal{A}(x)(\xi), \eta) & =\frac{1}{\left\langle A^{-1} x, A^{-1} x\right\rangle}\left\langle\xi-\frac{\langle x, \xi\rangle}{\left\langle A^{-1} x, x\right\rangle} A^{-1} x, \eta\right\rangle \\
=\frac{1}{\left\langle A^{-1} x, A^{-1} x\right\rangle}\langle\xi, \eta\rangle & =\frac{g_{x}(\xi, \eta)}{\left\langle A^{-1} x, A^{-1} x\right\rangle} .
\end{aligned}
$$

Therefore, we have proved that

$$
\left.\mathcal{A}(x)\right|_{T_{x} E_{h}}=\left(\frac{\Pi_{i=1}^{n} a^{i}}{h}\right)^{\frac{1}{n}} A\left(\left.g\right|_{E_{h}},\left.\bar{g}\right|_{E_{h}}\right) .
$$

Similarly, we have

$$
\begin{aligned}
g\left(\mathcal{A}^{\prime}(x)(\xi), \eta\right) & =\left\langle A^{-1} \xi-\frac{\left\langle A^{-2} x, \xi\right\rangle}{\left\langle A^{-1} x, A^{-1} x\right\rangle} A^{-1} x, \eta\right\rangle \\
& =\left\langle A^{-1} \xi, \eta\right\rangle=\left\langle A^{-1} x, A^{-1} x\right\rangle \bar{g}(\xi, \eta) .
\end{aligned}
$$

Hence,

$$
\left.\mathcal{A}^{\prime}(x)\right|_{T_{x} E_{h}}=\left(\frac{h}{\prod_{i=1}^{n} a^{i}}\right)^{\frac{1}{n}}\left[A\left(\left.g\right|_{E_{h}},\left.\bar{g}\right|_{E_{h}}\right)\right]^{-1} .
$$

Finally, it is obvious that if a self-adjoint operator is given on an invariant hyperplane, then the normal vector is an eigenvector and the corresponding eigenvalue determines the operator uniquely on the whole space. 
Theorem 6. Consider the family of Riemannian metrics $g^{(k)} \stackrel{\text { def }}{=}$ $\left\langle\mathcal{A}^{k}(x)(\xi), \eta\right\rangle$ and $\bar{g}^{(k)} \stackrel{\text { def }}{=} \frac{1}{\left\langle A^{-1} x, A^{-1} x\right\rangle}\left\langle\mathcal{A}^{k-1}(x)(\xi), \eta\right\rangle(k \in Z)$ on $R^{n} \backslash\{0\}$.

1) The restriction of $g^{(k)}$ to any ellipsoid $E_{h}$ gives a completely integrable Riemannian metric. The corresponding integrals are given by formula

$$
I_{\alpha, \beta}^{(k)} \stackrel{\text { def }}{=} \frac{\operatorname{det}(\alpha \mathcal{A}(x)+\beta)}{\alpha\left\langle A^{-1} x, A^{-1} x\right\rangle+\beta} \mathcal{A}^{k}(x)(\alpha \mathcal{A}(x)+\beta)^{-1},
$$

where $\alpha$ and $\beta$ are parameters.

2) The restriction of $\bar{g}^{(k)}$ to any ellipsoid $E_{h}$ gives a completely integrable Riemannian metric. The corresponding integrals are given by formula

$$
\bar{I}_{\alpha, \beta}^{(k)} \stackrel{\text { def }}{=} \frac{I_{\beta, \alpha}^{(k)}}{\left\langle A^{-1} x, A^{-1} x\right\rangle^{2}} .
$$

3) The metrics $g^{(k)}$ and $\bar{g}^{(k)}$ are contained in a 1-parameter family of metrics

$$
\bar{g}_{\alpha, \beta}^{(k)} \stackrel{\text { def }}{=} \frac{\alpha\left\langle A^{-1} x, A^{-1} x\right\rangle+\beta}{\operatorname{det}(\alpha \mathcal{A}(x)+\beta)} \mathcal{A}^{(k)}(x)(\alpha \mathcal{A}(x)+\beta)^{-1},
$$

and the restrictions of any two metrics from this family to the ellipsoid $E_{h}$ are geodesically equivalent.

The proof easily follows from Proposition 4, Theorem 4 and Theorem 5 .

The Ellipsoid and the Poisson sphere. In the case $k=1$ we obtain that the metrics

$$
\begin{aligned}
d g_{(1)}^{2} & =\langle\mathcal{A}(x) d x, d x\rangle \\
& =\langle A d x, d x\rangle-\frac{\langle x, d x\rangle^{2}}{\left\langle A^{-1} x, x\right\rangle}+\left\langle A^{-1} x, d x\right\rangle^{2}
\end{aligned}
$$

and

$$
d \bar{g}_{(1)}^{2}=\frac{1}{\left\langle A^{-1} x, A^{-1} x\right\rangle}\langle d x, d x\rangle
$$

are geodesically equivalent on the ellipsoid $E_{h}, h>0$.

The metric $\bar{g}^{(1)} \mid E_{h}$ can be considered as a metric on the homogeneous space $S^{n}=S O(n+1) / S O(n)$, where the kinetic energy of the $(n+1)$ dimensional rigid body is spread left-invariantly on the whole $S O(n+1)$. 
This construction generalize the well-known metric on the Poisson sphere considered in the classical mechanics (see $[\mathbf{1 0}]$ ).

Corollary 2. The restriction of the metric $d g_{(1)}^{2}$ to the ellipsoid $E_{h}$ has the same geodesic lines as the metric on the Poisson sphere.

Using Proposition 2, we obtain

Corollary 3. The geodesic flows on the ellipsoid and the Poisson sphere have identical Liouville foliations.

If $n=2$ the last result is well-known. Moreover, in [11] A. T. Fomenko and A. V. Bolsinov using the theory of orbital equivalence of the integrable Hamiltonian systems proved that for every 2-dimensional ellipsoid we can take a suitable Poisson sphere such that the corresponding geodesic flows are continuously orbitally equivalent. It is interesting to solve the same problem in multidimensional case.

The standard sphere and the Poisson sphere. In the case $k=-1$ we have

$$
\begin{aligned}
d g_{(-1)}^{2} & =\left\langle\mathcal{A}^{\prime}(x) d x, d x\right\rangle \\
& =\left\langle A^{-1} d x, d x\right\rangle-2 \frac{\left\langle A^{-1} x, d x\right\rangle\left\langle A^{-2} x, d x\right\rangle}{\left\langle A^{-1} x, A^{-1} x\right\rangle} .
\end{aligned}
$$

The last term on the right side vanish on $T E_{h}$. Therefore, we can think that $d g_{(-1)}^{2}=\left\langle A^{-1} d x, d x\right\rangle$. Changing the variables $x=\sqrt{A} y$ we see that $g_{(-1)}$ is the standard metric on the sphere. Further,

$$
d \bar{g}_{(-1)}^{2}=\frac{\left\{\left\langle A^{-1} d x, A^{-1} d x\right\rangle-\frac{\left\langle A^{-1} x, A^{-1} d x\right\rangle^{2}}{\left\langle A^{-1} x, A^{-1} x\right\rangle}\right\}}{\left\langle A^{-1} x, A^{-1} x\right\rangle} .
$$

Corollary 4. The restriction of the metric

$$
d \bar{g}_{(-1)}^{2}=\frac{\left\langle A^{-1} d y, d y\right\rangle-\frac{\left\langle A^{-1} y, d y\right\rangle^{2}}{\left\langle A^{-1} y, y\right\rangle}}{\left\langle A^{-1} y, y\right\rangle}
$$

to the sphere $S^{n}=\{\langle y, y\rangle=h>0\}$ has the same geodesic lines as the standard metric on the sphere. 
Using Proposition 3, we obtain

Corollary 5. The metric on the Poisson sphere $\left.d \bar{g}_{(1)}^{2}\right|_{E_{h}}$ and the standard metric on the sphere $\left.d g_{(-1)}^{2}\right|_{E_{h}}$ are completely integrable on $\mathcal{T}^{*} M$ such that the corresponding integrals are the same.

\section{A family of integrable Hamiltonians in $e(3)^{*}$}

It is well-known that the Euler-Poisson equations which describe the motion of the rigid body can be written as Euler equations on the dual space of the Lie algebra $e(3)$ (see [12], [13]). More precisely, if the coordinates in $e(3)^{*}$ are denoted by $\left(r_{1}, r_{2}, r_{3}, s_{1}, s_{2}, s_{3}\right)$, then the LiePoisson bracket can be given by relations $\left\{s_{i}, s_{j}\right\}=\epsilon_{i j k} s_{k},\left\{r_{i}, r_{j}\right\}=0$, $\left\{s_{i}, r_{j}\right\}=\epsilon_{i j k} r_{k}$. Therefore, if $H=H(r, s)$ is a Hamiltonian function, then the Euler equations take the form of Kirchoff equations

$$
\begin{aligned}
& \dot{s}=s \times \frac{\partial H}{\partial s}+r \times \frac{\partial H}{\partial r}, \\
& \dot{r}=r \times \frac{\partial H}{\partial s} .
\end{aligned}
$$

The Lie-Poisson bracket has two annihilators $F_{1}=r_{1}^{2}+r_{2}^{2}+r_{3}^{2}$ and $F_{2}=r_{1} s_{1}+r_{2} s_{2}+r_{3} s_{3}$. The Hamiltonian function for the rigid body is

$$
H \stackrel{\text { def }}{=} \frac{1}{2}\left(\frac{s_{1}^{2}}{I_{1}}+\frac{s_{2}^{2}}{I_{2}}+\frac{s_{3}^{2}}{I_{3}}\right)+m g\langle l, r\rangle,
$$

where $s=\left(s_{1}, s_{2}, s_{3}\right)$ denotes the angular momentum of the body, $l=\left(l_{1}, l_{2}, l_{3}\right)$ denotes the coordinates of the center of gravity of the body, $I=\operatorname{diag}\left(I_{1}, I_{1}, I_{3}\right)$ is the tensor of inertia, $r=\left(r_{1}, r_{2}, r_{3}\right)$ - the coordinates of the unit vertical vector in the space, and $m g$ is the weight of the body.

The cotangent bundle $T^{*} S^{2}$ supplied with the canonical symplectic structure $d p \wedge d q$ is symplectomorphic to the manifold $\mathcal{O}_{h} \stackrel{\text { def }}{=}\left\{F_{1}=\right.$ $\left.h, F_{2}=0\right\} \subset e(3)^{*}$ (see [14]). Therefore, any integrable Hamiltonian on $T^{*} S^{2}$ gives an integrable Hamiltonian on $\mathcal{O}_{h}$. Moreover, if the first Hamiltonian is polynomial in impulses, then the second one is also polynomial in momenta $s=\left(s_{1}, s_{2}, s_{3}\right)$ and has the same degree. Using the results of $[\mathbf{1 4}]$ and Lemma 2 it is easy to prove 
Lemma 3. Suppose that the Riemannian metric $d g^{2}=g_{i j} d x^{i} d x^{j}, x=$ $\left(x^{1}, x^{2}, x^{3}\right)$ is smooth in a neighborhood of the sphere $S_{h}^{2}=\{\langle x, x\rangle=h\}$ and the restriction $\left.d g^{2}\right|_{S_{h}^{2}}$ gives a metric whose geodesic flow is completely integrable; then the Hamiltonian function

$$
\mathcal{H}_{g}(r, s) \stackrel{\operatorname{def}}{=}\left[\frac{\operatorname{det} E}{\operatorname{det} G} \frac{1}{e\left(n_{e}, n_{g}\right)}\right] \sum_{i, j} g_{i j}(r) s_{i} s_{j},
$$

where $d e^{2}=\left(d x^{1}\right)^{2}+\left(d x^{2}\right)^{2}+\left(d x^{3}\right)^{2}, G=\left(g_{i j}\right), E=\left(e_{i j}\right)$ and $n_{e}$ and $n_{g}$ are the unit external normal vectors to the sphere $S_{h}^{2}$ calculated with respect to the metrics $e$ and $g$ respectively at the point $x=r \in S_{h}^{2}$, is completely integrable on the submanifold $\mathcal{O}_{h}$.

In Section 4 we found a family of metrics on $R^{n}$ such that their restriction on the ellipsoid $E_{h}$ give a family of integrable metrics. Now, using these metrics and Lemma 3 we are able to find a family of integrable Hamiltonians in $e(3)^{*}$.

Theorem 7. Consider the operators

$$
\mathcal{A}(r) \stackrel{\text { def }}{=} A-\frac{(\sqrt{A} r) \otimes(\sqrt{A} r)}{\langle r, r\rangle}+\left(A^{-\frac{1}{2}} r\right) \otimes\left(A^{-\frac{1}{2}} r\right)
$$

and

$$
\mathcal{B}^{(k)}(r) \stackrel{\text { def }}{=} \sqrt{A} \mathcal{A}^{k}(r) \sqrt{A} .
$$

For any fixed integer $k$ the Hamiltonians

$$
H^{(k)} \stackrel{\text { def }}{=} \frac{1}{\left\langle A^{-1} r, r\right\rangle^{k+1}}\left\langle\mathcal{B}^{(k)} s, s\right\rangle
$$

and

$$
\bar{H}^{(k)} \stackrel{\text { def }}{=} \frac{1}{\left\langle A^{-1} r, r\right\rangle^{k-1}}\left\langle\mathcal{B}^{(k-1)} s, s\right\rangle
$$

are Liouville integrable on any orbit $\mathcal{O}_{h}$. Moreover, the corresponding Hamiltonian systems are orbitally equivalent.

Example. If $k=1$, then we obtain that the Hamiltonians

$$
H^{(1)}=\frac{1}{\left\langle A^{-1} r, r\right\rangle^{2}}\left\{\left\langle A^{2} s, s\right\rangle-\frac{\langle A r, s\rangle^{2}}{\langle r, r\rangle}+\langle r, s\rangle^{2}\right\}
$$

and

$$
\bar{H}^{(1)}=\langle A s, s\rangle
$$

are orbitally equivalent. 
Corollary 6. The Hamiltonian system with Hamiltonian $H^{(1)}$ and the Euler case of the free motion of the rigid body are orbitally equivalent on the orbit $\mathcal{O}_{h}, h>0$.

\section{References}

[1] P. J.Topalov, Tensor invariants of natural mechanical systems on compact surfaces, and the corresponding integrals, Mat. Sb. 188(2) (1997), 137-157.

[2] P. J. Topalov and V. S. Matveev, Geodesic equivalence and integrability, Preprint of MPI-Bonn (1998), MPI 98-74.

[3] V. S. Matveev and P. J. Topalov, Geodesic equivalence and Liouville integrability, Regular and Chaotic Dynamics 2 (1998), 29-44.

[4] S. V. Bolotin and V. V. Kozlov, Symmetry fields of geodesic flows, Russian J. Math. Phys. 3(3) (1995), 279-295.

[5] P. Painlevé, Sur les intégrale quadratiques des équations de la Dinamique, C. R. Acad. Sci. Paris Sér. I Math. 124 (1897), 221-224.

[6] T. Levi-Civita, Sulle trasformazioni delle equazioni dinamiche, Ann. Mat. (2) 24 (1896), 255-300.

[7] A. V. Aminova, Pseudo-Riemannian manifolds with general geodesics, Uspekhi Mat. Nauk 48(2) (1993), 107-164 (in Russian); translation in Russian Math. Surveys 48(2) (1993), 105-160.

[8] N. S. Sinyukov, To the theory of geodesical mappings, Soviet Math. Docl. 169(4) (1966), 770-772.

[9] S. Tabachnikov, Projectively equivalent metrics, exact transverse line field and the geodesic flow on the ellipsoid, Comment. Math. Helv. 74(2) (1999), 306-321.

[10] A. V. BRAILOV, Construction of completely integrable geodesic flows on compact symmetric spaces, Math. USSR Izvestiya 29(1) (1987), 19-31.

[11] A. V. Bolsinov And A. T. Fomenko, Orbital classification of geodesic flows of two-dimensional ellipsoids. The Jacobi problem is orbitally equivalent to the integrable Euler case in the dynamics of a rigid body, Funktsional. Anal. i Prilozhen 29(3) (1995), 1-15, 96 (in Russian); translation in Funct. Anal. Appl. 29(3) (1995), 149-160.

[12] S. P. Novikov and I. Shmel'tser, Periodic solutions of Kirchhoff equations for the free motion of a rigid body in a fluid and the extended Lyusternik-Shnirel'man-Morse theory, Funktional. Anal. $i$ Prilozhen. 15(3) (1981), 54-66 (in Russian). 
[13] V. V. Kozlov, Two integrable problems of classical dynamics, Vestnik Moskov. Univ. Ser. I Mat. Mekh. 4 (1981), 80-83, 87 (in Russian).

[14] A. V. Bolsinov, A. T. Fomenko and V. V. Kozlov, The de Maupertuis principle and geodesic flows on a sphere that arise from integrable cases of the dynamics of a rigid body, Uspekhi Mat. Nauk 50(3) (1995), 3-32 (in Russian); translation in Russian Math. Surveys 50(3) (1995), 473-501.

Department of Differential Equations

Institut of Mathematics and Informatics

Bulgarian Academy of Sciences

Acad. G. Bonchev Str., bl. 8

Sofia 1113

Bulgaria

E-mail address: topalov@math.bas.bg

Primera versió rebuda el 30 d'abril de 1999, darrera versió rebuda el 23 de setembre de 1999. 